\title{
Multilinguales
}

\section{Printemps arabe : de l'événement à la formule discursive}

Arab spring : from the event to the discursive formula

\section{Nadjia Nehari-Roubai et Farid Benramdane}

\section{(2) OpenEdition}

\section{Journals}

Édition électronique

URL : http://journals.openedition.org/multilinguales/3580

DOI : $10.4000 /$ multilinguales.3580

ISSN : 2335-1853

Éditeur

Université Abderrahmane Mira - Bejaia

Référence électronique

Nadjia Nehari-Roubai et Farid Benramdane, «Printemps arabe : de l'événement à la formule discursive », Multilinguales [En ligne], 10 | 2018, mis en ligne le 31 décembre 2018, consulté le 30 mai 2020. URL : http://journals.openedition.org/multilinguales/3580 ; DOI : https://doi.org/10.4000/ multilinguales.3580

Ce document a été généré automatiquement le 30 mai 2020.

\section{(c) $(1) \Theta$}

Multilinguales est mise à disposition selon les termes de la Licence Creative Commons Attribution Pas d'Utilisation Commerciale - Pas de Modification 4.0 International 


\title{
Printemps arabe : de l'événement à la formule discursive
}

\author{
Arab spring : from the event to the discursive formula
}

Nadjia Nehari-Roubai et Farid Benramdane

1 À certains moments-clés de l'histoire d'un pays, d'une partie du monde, d'une façon assez soudaine et en même temps d'une façon qui semble évidente, surgissent des mots, des syntagmes, une phraséologie qui rentrent rapidement dans le débat public et le champ de la communication sociale. Ces expressions se trouvent happées au passage et propagées par les médias devenant ainsi des concepts "à la mode ", prisés par les discours publics et politiques qui ne leur attribuent pourtant pas toujours les mêmes définitions. Ainsi, leur circulation foisonnante ajoutée à leur flou sémantique, font de ces émergences discursives des « formules » dans le discours.

2 C'est le cas des expressions " " Printemps arabe ", "Printemps des peuples arabes " " Printemps égyptien " ou encore "révolution du jasmin». Une floraison d'expressions printanières qui, pour désigner les différentes révoltes populaires qui ont ébranlé le monde arabe depuis la fin de l'année 2010, ont éclos, ont germé et se sont " figées » pendant une certaine période dans le paysage social, véhiculées " pour le meilleur et pour le pire " par la télévision, les réseaux sociaux ${ }^{1}$, les discours politiques et la presse.

Facilité journalistique et référence historique sont les arguments avancés par les journalistes pour justifier cette création lexicale. Il est vrai, d'une part, que le recours aux effets de style telle que la métaphore est une pratique facile et courante dans l'écriture journalistique et c'est justement parce que les révolutions ont pour but un " réveil », qu'on les compare au printemps. D'autre part, l'expression " printemps arabe »(désormais PA) est aussi choisie par effet de rapprochement historique avec d'autres printemps de l'Histoire : notamment avec Le Printemps des peuples en Europe en 1848 et Le Printemps de Prague de Janvier 1968. Le rapprochement semble tout à fait légitime vu que ces printemps comme le PA ont pour événement commun " la révolution des peuples » et le désir de changements politiques et sociaux dans leurs pays respectifs, ce qui autoriserait les journalistes à utiliser cette dénomination, laquelle passerait inaperçue dans les médias. 
Mais à quoi réfère l'expression PA ? Comment s'est-elle imposée comme formule dans les discours ? La réalité à laquelle renvoie alors PA s'avère multiple et très controversée. Une réalité étroitement associée à la prise en compte des enjeux de diverses natures : politiques, culturels, identitaires...

Dans notre article, nous tentons d'analyser cette expression que, nous pensons ne saurait s'en tenir à de simples considérations d'ordre stylistique ou à des mises en parallèle historiques. Plus précisément, nous souhaitons vérifier dans quelle mesure une formule chargée d'enjeux de diverses natures comme PA peut permettre à ses utilisateurs de se positionner politiquement, et de façon implicite dans l'énoncé, face à un événement historique d'une telle ampleur. Notre objet n'est pas l'événement luimême mais sa construction discursive, analysée à travers la formule.

\section{«Printemps arabe » : une formule figée pour un événement instable}

6 L'objectif de notre recherche est d'interroger la notion de PA et suivre son destin de formule lié à l'ordre événementiel et à son interprétation à travers l'espace discursif auquel elle participe. Et c'est à Alice Krieg-Planqué (2003 et 2009) que nous devons la théorisation et conceptualisation de la notion de " formule ». Une notion qui a été repensée $^{2}$ rigoureusement dans ses travaux sur l'histoire et l'usage de la formule purification ethnique. Krieg lie la notion de formule à la nomination d'un événement historique qui en détermine l'émergence et la large diffusion dans la sphère publique. La linguiste montre que dans un premier temps, la formule émerge sous la forme de différentes proto-formules qui donnent lieu ensuite à la stabilisation d'une forme figée. Outre son figement linguistique, les caractéristiques principales de la formule, sont : sa vaste circulation; sa capacité de fonctionner comme synthèse d'un contenu conceptuel ; le fait d'être l'objet de commentaires métalinguistiques, méta-énonciatifs et de nature polémique sur le positionnement idéologique que la formule est susceptible de condenser (Krieg, $2009: 63$ ).

7 Pour notre part, nous comptons dégager le fonctionnement de la séquence PA en tant que formule à partir d'un corpus de discours médiatisés assez complexe et hétérogène, articulé sur deux types de supports. Le premier, déterminant pour notre analyse, correspond à la presse écrite et est composé d'articles publiés dans des quotidiens français et algériens d'expression française (Le Monde, Libération, Le Figaro, El Watan, le Quotidien d'Oran,) datant des années 2011 à 2015. Il n'est pas question de corpus de texte (article) mais plutôt un corpus d'énoncés (titre, chapeau ou quelques passages de l'article) où figure la formule. Notre deuxième corpus, plutôt hétéroclite, réunit des extraits d'articles tirés de revues spécialisées (revues d'histoire, de géopolitique, de sociologie...), des blogs qui portent sur les révolutions du monde arabe (2011 à 2015) et une série de caricatures. L'exploration de ce type de corpus nous permettra de témoigner de la circulation de la séquence étudiée sous des formes discursives diversifiées.

8 Ainsi, et pour rendre compte des phénomènes d'inscription de la formule PA, nous analyserons et tenterons de déterminer les conditions socioculturelles et sociopolitiques justifiant son apparition (sa genèse). Dans un deuxième temps, nous 
inspecterons ses propriétés telles que les propose Krieg et enfin, nous relèverons le caractère de la mise en circulation de la formule PA à travers notre corpus.

\section{Émergence de la formule «printemps arabe »}

Repérer le tout premier usage de la formule PA est une tâche assez ardue. Reconstruire le processus de transformation de la séquence en formule l'est encore plus. Nous tentons, toutefois d'appréhender son apparition et sa mutation dans la place publique grâce à l'analyse de notre corpus.

10 L'expression PA fera son apparition dans les champs médiatiques, politiques et publics dès le début du mouvement protestataire populaire parti de Tunisie et poursuivant son extension vers d'autres pays du " monde arabe » depuis l'Égypte et la Libye jusqu'au Yémen et Bahreïn. En effet, le 17 décembre 2010, Mohamed Bouazizi, un vendeur de fruits et légumes, s'auto-immole en Tunisie, pour dénoncer des politiques sociales restreintes par son gouvernement. Il sera l'élément déclencheur des vagues de soulèvements inédites dans le "monde arabe ». Entre 2011 et 2013, trois gouvernements vont tomber : celui de Ben Ali en Tunisie, de Moubarak en Égypte et de Kadhafi en Libye. Sur la péninsule arabique, la contestation se fait plus timide. Mais c'est en Syrie que la situation est la plus alarmante car le pays est, encore aujourd'hui, en pleine guerre. D'un bout à l'autre du " monde arabe ", de l'Atlantique jusqu'au Golfe persique, le "monde arabe " devient alors le théâtre d'une succession de soulèvements populaires de grande ampleur comparés hâtivement aux révolutions européennes du milieu du XIXe siècle connues sous l'expression lyrique « printemps des peuples ».

11 Mais ce n'est pas la première fois que l'expression PA éclot dans l'espace public. Selon M.Camau (2012), son usage figurait déjà dès la fin des années 1950, dans les carnets de voyage d'un journaliste-historien, Benoist-Méchin, ancien secrétaire d'État du gouvernement de Vichy et passionné de l'Orient. En février 2005, George W. Bush avait employé le terme pour référer à la floraison de courte durée des mouvements démocratiques au Moyen- Orient avec les premières élections en Irak et les manifestations au Liban. Le choix de l'expression témoigne déjà du souhait de voir en ces événements des révolutions, rappelant que pour le président américain, la promotion de la démocratie représentait alors l'axe principal de la politique américaine. Cependant, ces tentatives ont pris un autre tournant : invasion de l'Irak en 2003, révolution du Cèdre en 2005, soutenue par les pays occidentaux, et guerre du Liban de 2006. L'expression PA sera utilisée trois ans plus tard, au cours de l'année 2008, par certains penseurs comme Burhan Galion quand il a décrit les transformations démocratiques, qui ont été prévues au début de troisième millénaire, par le " printemps arabe ". Il dira à ce propos qu'il s'agit là d'« un printemps qui n'a pas été accompli » (Lugan, 2011). Il faudra attendre 2011, pour que les médias s'« emballent $\aleph^{3}$ et s'accordent sur l'expression PA afin de qualifier les différents soulèvements dans le monde arabe.

12 Ainsi, "L'acte de nommer des événements dans les médias semble banal, parce que naturel : on a besoin d'un nom pour les décrire, les identifier, bref pour en parler » dira Calabrese Steimberg (2013: 233). Krieg (2012) parle, elle, de la catégorisation un peu rapide par les journalistes pour expliquer les événements à leur lectorat. Par un besoin médiatique, la presse peut utiliser n'importe quelle dénomination et classification 
qu'elle juge pertinente pour désigner un événement sans le consensus social. Pourtant, le public, lui aussi, peut participer à l'acte de nommer l'événement historique, le cautionner ou le réfuter notamment s'il l'a vécu. Suivre l'événement dès qu'il surgit, le commenter, l'influencer devient possible aujourd'hui pour les locuteurs-auditeurs grâce en particulier au web 2.0. "Le processus des commentaires et des conflits d'intérêts devient (alors) visible »(Calabrese Steimberg, 2013 : 233). L'exemple le plus explicite, pour rester dans notre contexte est celui de la dénomination " révolution du jasmin » qui a été proposé par les médias pour renvoyer à la révolution de 2010-2011 en Tunisie. Encore une fois l'expression fait référence à " la révolution des œillets " qui réfère à la révolution portugaise de 1974. Cette expression n'avait alors pas trouvé un écho favorable auprès du peuple tunisien qui a contesté sa justesse et l'a ressentie comme une moquerie sur la nature de la révolution qui en fait était à ses yeux une révolution de la liberté et de la dignité4. Les Tunisiens, journalistes, internautes n'ont pas manqué alors de montrer leur indignation face aux médias étrangers leur refusant le droit de nommer leur révolution personnelle. La polémique sur le choix de la nomination d'un événement est d'ailleurs une des propriétés qui élève un mot au rang de formule en discours.

\section{Les propriétés de la formule « printemps arabe »}

13 Nous considérons que la séquence PA a fonctionné dès sa réapparition (2011) et continue à fonctionner comme une formule chargée d'enjeux multiples dans les espaces publics dans lesquels elle circule. Alice Krieg nous propose de circonscrire alors " l'objet formule à travers ses propriétés, en tant que celle-ci : présente un caractère figé, s'inscrit dans une dimension discursive, fonctionne comme un référent social, comporte un aspect polémique » (Krieg, 2009 : 65).

\section{"Printemps arabe »: une séquence figée}

La première condition pour parler de formule correspond au figement du signifiant : « la formule est portée par une forme signifiante relativement stable " (Ibid : 65), qui la rend reconnaissable au grand public et qui permet sa répétition. En effet, l'usage récurent de l'expression PA dans notre corpus confirme son figement. De plus, chacune des unités composant cet ensemble ne peut fonctionner seule pour renvoyer au sens admis par tous. L'expression PA semble faire bloc et est connue de tous dans sa forme cristallisée. Elle est perçue comme un tout et « fonctionne comme une dénomination ». Toutefois la stabilité du signifiant n'est, en fait, que théorique car, la formule admet un certain nombre de variantes: celles-ci peuvent correspondre à des séquences qui reviennent à peu près dans les mêmes contextes : nous pensons notamment à printemps démocratique arabe, révolutions arabes, tsunami révolutionnaire arabe. Voici quelques exemples :

Vers un printemps démocratique arabe ? (Le Monde 15/01/2011)

Obama se disqualifie aux yeux des révolutions arabes naissantes. (Q.d'O Février 2011)

La Révolution arabe de 2011: à la recherche du sens perdu. (Confluences Méditerranée 2011/2 $n^{\circ} 77$ ).

Le roi tente de prémunir son pays du tsunami révolutionnaire arabe (Q.d'O du 25/26 février 2011) 
$\grave{A}$ travers ces quelques exemples, on retrouve l'aspect formulaire de ces expressions formant une phraséologie du PA.

\section{"Printemps arabe » : une dimension discursive}

Comme le rappelle Krieg, même si elle est supportée par une matérialité linguistique stable qui la rend repérable, la formule n'est pas une notion linguistique. L'unité d'observation d'une formule n'est pas la phrase, mais le discours, comme ensemble de productions langagières situées. La formule s'inscrit dans une dimension discursive : « la formule n'existe pas sans les usages qui la font advenir comme telle » (Krieg, 2009: 84).

Concernant PA, nous pouvons avancer que la séquence est inscrite dans une dimension discursive en tant que formule : c'est à travers ses nombreux emplois dans toutes sortes de discours (articles, émissions radiophoniques ou télévisées, discours, réseaux sociaux) que cette séquence s'est figée grâce à ses acteurs sociaux desquels dépendent sa légitimation ou sa disqualification en tant que formule (Krieg, 2003 : 14).

\section{«Printemps arabe »: un référent social}

Même si ses significations sont multiples et contradictoires, la formule fonctionne comme un référent social : en effet, le public de toute origine et dans toutes les langues emploie la séquence PA dans des discours variés oraux et écrits allant de la presse à la conversation ordinaire, sans qu'il y ait besoin d'expliquer de quoi il s'agit, ce qui laisse penser à un consensus sur ce à quoi renvoie PA. " La formule réfère : elle renvoie au monde, elle « évoque quelque chose pour tous à un moment donné » (Krieg, 2009: 95). Le signe acquiert alors une "notoriété » et parmi les indices qui permettent de dire que la formule est supposée référer, nous citons les diverses formes de présupposé.

Par exemple : L'article défini le qui inscrit le syntagme dans le " déjà su " et le " déjà connu »: le printemps arabe. Ou encore, dans les titres de presse, le renvoi au monde qui se fait par la structure $\mathrm{X}$ deux points $\mathrm{Y}$ :

Printemps arabe : quelles conséquences pour le Maroc ? (Le Monde 09/03/2011)

Printemps arabe : pas de démocratie sans les femmes. (Le Monde 30/03/2011)

Printemps arabe : réussir la révolution économique. (Le Monde 18/04/2011)

Autre caractère notoire de la formule (référent), c'est la productivité lexicologique. En effet les créations lexicales laissent croire que le " signe de base » est suffisamment connu du lecteur-interlocuteur, " quand bien même ce signe de base avance grimé ou masqué » (Krieg 2009 : 97). Voici quelques exemples de cette productivité lexicologique basée notamment sur le défigement et le jeu de mots :

Du jour au lendemain, le paysage politique algérien s'est déverrouillé le printemps des nouveaux partis (El Watan 23/04/2012).

L'hiver des dictateurs (le Q.d'O 03/02/2011)

Arabités numériques. Le printemps du Web arabe (Actes Sud 2012)

Les fruits verts $\mathrm{du}$ "printemps arabe"(Le Monde 02/06/2011) des pays arabes " printanisés "... (ahmedbenssaada.com 22/01/2016)

Ou le calembour dans les caricatures qui suivent : 


\section{PRINTEMPS ARABE PRINTEMPS À BARBE}
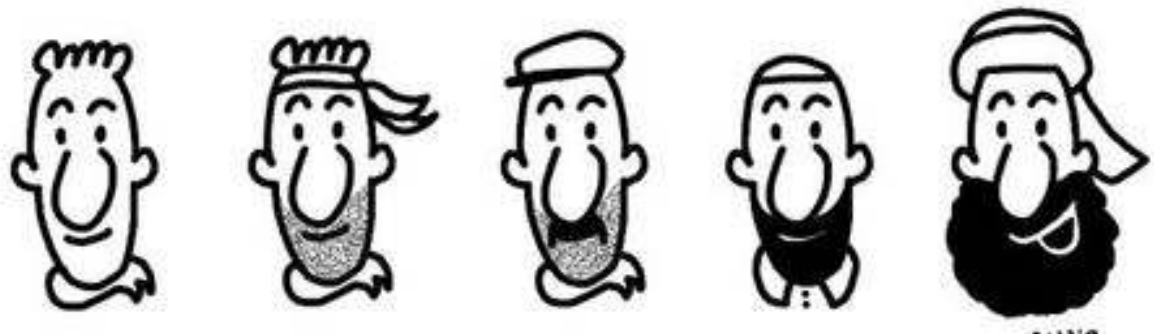

\section{Silence, ça pousse!}

autio

14)

chribactu.20minutes-blogs.f30/01/2013

\section{CALLANETBOUR!}

\section{PRINTETPS ARABE}

ANTOTNE À ROBE
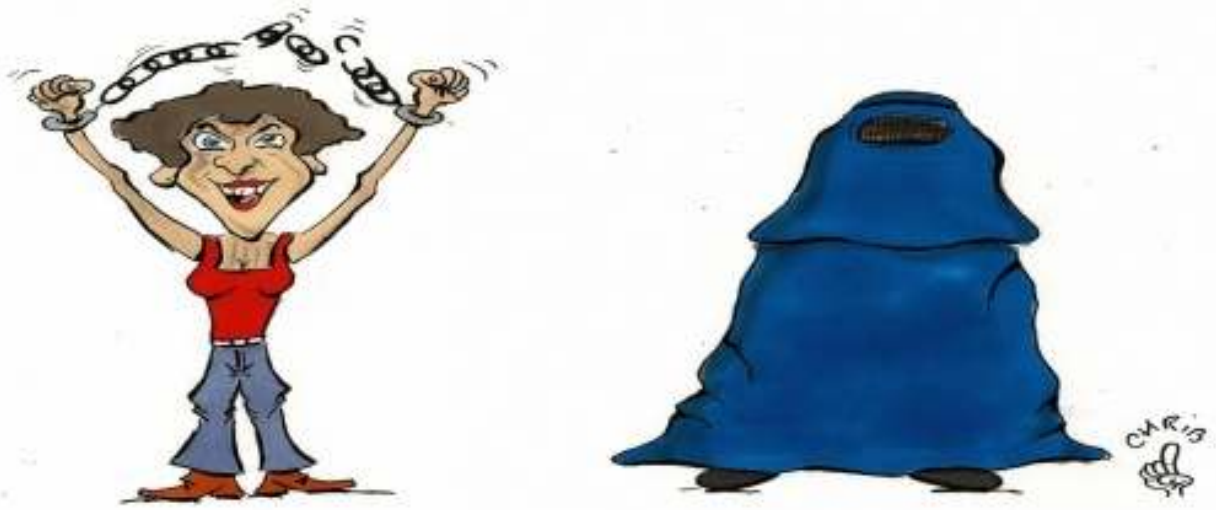

Ou encore le recours au mot-valise comme avec « arabellion » en titre d'article construit par l'association de l'item arabe et celui de rébellion:

15)Arabellion (Michel Korinman, in revue Outre-terre, 2011/3, $n^{\circ} 29$ )

« Printemps arabe »: un printemps embrumé

20 La formule a une dimension polémique : Krieg définit la formule comme " un ensemble de formulations qui, du fait de leurs emplois à un moment donné et dans un espace public donné, cristallisent des enjeux politiques et sociaux que ces expressions 
contribuent dans le même temps à construire. » (Ibid: 7). En effet, si la formule PA, comme nous l'avons constaté précédemment " évoque quelque chose pour tous " de par son figement et son caractère de référent social, il n'en reste pas moins que celle-ci est aussi récusée et s'inscrit, à l'évidence, dans une dimension proprement polémique puisqu'elle constitue un enjeu socio-politique. La formule PA et ses variantes ne cessent d'être remises en question. Les supports médiatiques, les articles scientifiques, les réseaux sociaux, les blogs emploient la formule PA en tant qu'attribut pour nommer les événements qui ont éclaté en 2011 dans cette partie du monde, en soulignant souvent la nature problématique et les enjeux que cette expression cache. Le signifiant PA est alors souvent « mis en cause " par des guillemets ou en italique :

On a vu ces révoltes dans ce qu'on a appelé trop vite "le printemps arabe ". (Samir Amin dans le blogue socialgerie 11/02/2012)

Remarques sur ledit "printemps arabe"(Salah Sakhri dans alterinfo.net.17/05/2013)

Comment analyser la situation politico-religieuse issue de ce qu'il est convenu d'appeler le "printemps arabe" ? (Pierre Lafrance, le Monde 09/01/2012)

L'usage des guillemets, précédés comme dans les exemples ci-dessus, des expressions (ce qu'on a appelé, sur le dit, l'ambiguité du terme, ce qu'il est convenu d'appeler) témoignent explicitement, d'une prise de distance de la part de l'énonciateur par rapport à la séquence elle-même. Ils sont une marque de dialogisme interdiscursif (Moirand 2007 ; Krieg 2012 : 189) en ce qu'ils soulignent la circulation de la séquence d'un discours à l'autre et contribuent à lui attribuer, de ce fait, le statut de formule. Cette prise de distance avec la formule PA s'explique pour la plupart des énonciateurs par le refus d'assimiler ces mouvements contestataires de 2011 à la Révolution française. Assimilation largement saluée par certains journaux français comme Le Monde dans son édition du 16-17 janvier 2011 qui titrait " Révolution à la française au coeur du Maghreb » et ajoutait plus loin " une première poussée démocratique dans le monde arabe postcolonial. " De même, la couverture du journal Libération du 15 janvier 2011qui ne cachait pas son enthousiasme et permettait de trouver enfin, sur un journal français, l'image d'une femme arabe non voilée, manifestant et protestant de l'intérieur du monde arabe, avec un mot écrit en arabe, "El Houriya » (Liberté) ce qui deviendra plus tard un slogan des révolutions arabes avec l'expression « dégage ».

La Une de Libération du 15 janvier 2011

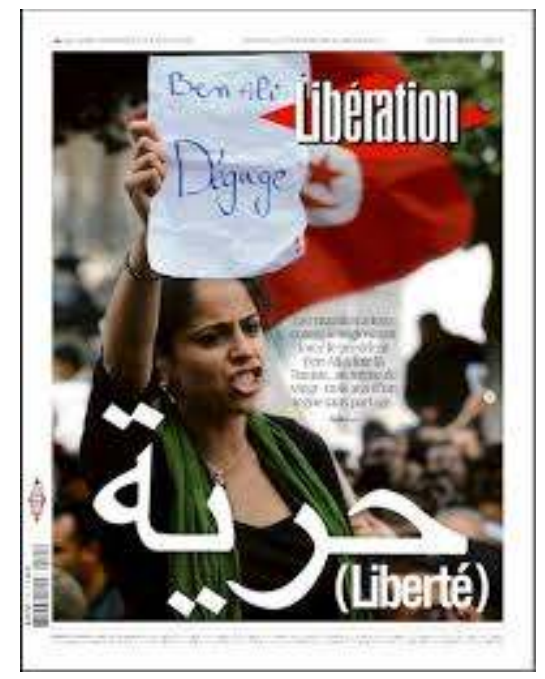


Ce rapprochement historique avec la Révolution française vu comme un héritage colonial n'est pas du goût de tout le monde. C'est le cas des Tunisiens - qui ne manquent pas de rappeler qu'ils ont été seuls à faire leur révolution- rejoints par plusieurs historiens et journalistes qui contestent le choix trop hâtif de l'expression PA jugée inappropriée et " très stéréotypée »:

"Printemps arabe": expression journalistique et surtout occidentale, orientaliste. Tragique illusion car l'expression est trompeuse: aucun lien entre les événements. (Printemps arabe - histoire d'une tragique illusion, lebonnetdespatriotes.net, 10/09/2014)

Le thème sur le Printemps arabe est, dès le départ, une allégeance et une soumission intellectuelle à la doxa occidentale qui, la première, a décidé d'appeler ainsi ces mouvements. (Chems Eddine CHITOUR. legrandsoir.info, 29/09/2011)

« printemps arabe », appellation plutôt usurpée (...) l'expression « printemps arabe » n'est pas un concept arabe, mais occidental. (M. Raimbaud in blog tunisie-secret.overblog.com, 03/03/2015).

Pour certains caricaturistes, le choix du mot « printemps » devrait être reconsidéré

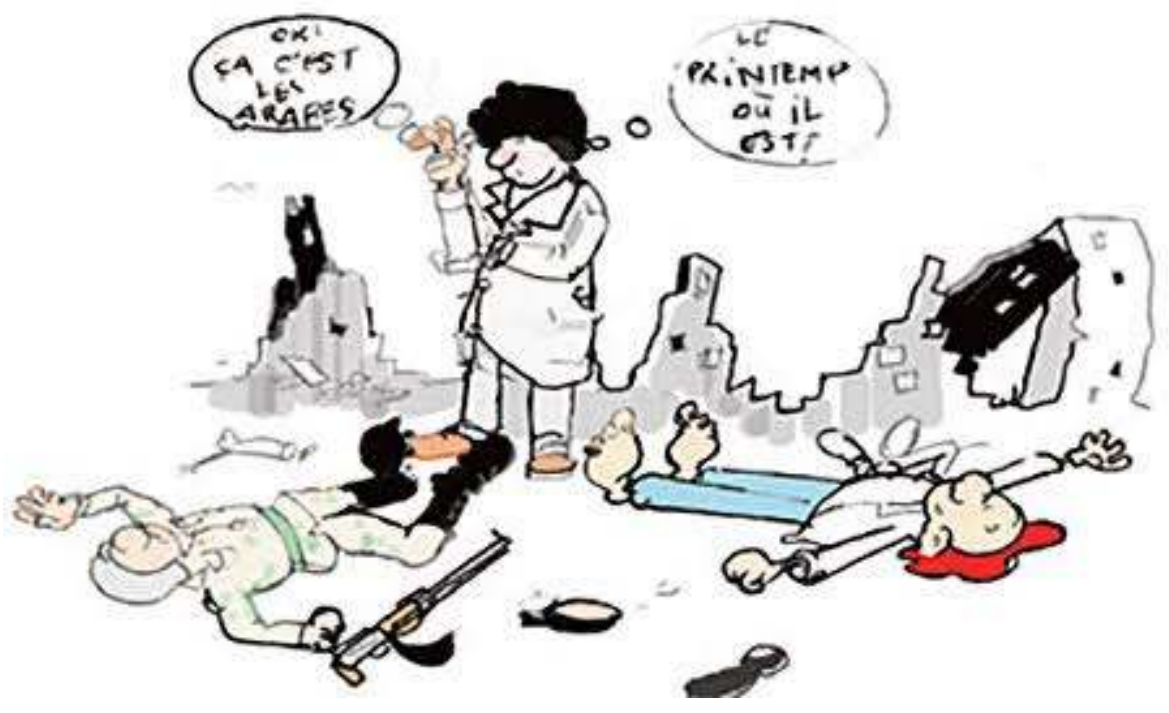




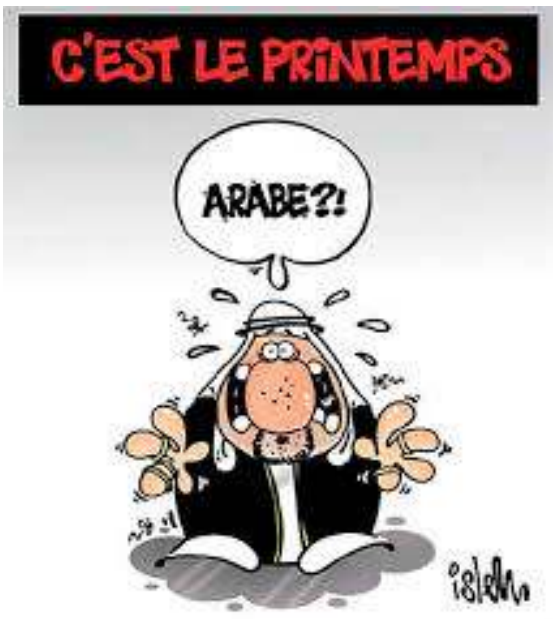

Même si quelques Tunisiens, à l'instar du journaliste Akram Belkaid, assument et revendiquent l'appellation de PA pour qualifier leur mouvement, précisant qu'elle a le mérite de faire accéder leur pays à la dignité des autres protagonistes de l'histoire universelle :

"L'expression fait référence au "printemps des peuples » de 1848, à des valeurs universelles, comme celles de la Révolution française de 1789 auxquelles nous, arabes, avons aussi droit d'accéder. On se révolte pour notre dignité, on chasse un tyran, cela dépasse les nationalités " ("Pourquoi parle-t-on de printemps des peuples arabes ?", Slate. fr, 22/02/2011).

C'est, enfin, au nom de leur droit à être reconnus comme des acteurs à part entière d'une histoire qui dépasse les particularismes, y compris en choisissant, selon leurs besoins du moment, des références à l'histoire française (Ben Brik, des mots pour seules armes ", L'Humanité, 18/07/2011)

Le caractère polémique de la formule PA se manifeste aussi par ce flou sémantique qu'on lui attribue. D'abord dans le sens accordé au mot " printemps ", ici, celui de " révolution ». Depuis les débuts du déclenchement du PA, tout le monde n'est pas d'accord pour dire s'il s'agissait de révolution, de révolte, de guerre civile ou de rébellion? Pour s'écarter de la métaphore saisonnière, il est vrai que certains ont préféré de loin le terme de révolution ou celui de révolte pour qualifier ces différentes manifestations. En arabe, les deux mots se disent Thawra qui signifie révolution, " ce que l'on entendait dans la rue " (Laurens H., Libération 10/08/2013). Ce mot est-il justifié ? La réponse est non pour beaucoup, car si comme le soutient Samuel Huntington la révolution consiste en " un changement interne rapide, fondamental et violent dans les valeurs dominantes et les mythes d'une société, dans ses institutions politiques, la structure sociale, le leadership, l'activité gouvernementale et les politiques $»^{5}$. Pour ces pays du PA, il n'y a pas eu de mouvement de révolution, de réforme, pas de réels objectifs, ni changements politiques et sociaux, encore moins de transition démocratique réelle :

Mais tout cela n'annonce pas nécessairement pour autant une révolution, soit un bouleversement dramatique du genre de ce qui s'est produit dans la France de 1789, pour la Russie à partir de 1919, voire en Iran avec la Révolution islamique de 1979, alors que des renversements violents de régime s'accompagnaient de changements profonds sur les plans social et culturel. Pour le moment, la révolte arabe tient plus de la 
rébellion politique que de la révolution historique (...). (Amotz Asa-el, En attendant la révolution, 2011)

(...) Qu'est-ce qui a été obtenu avec le départ des dictateurs ? La situation économique et sociale s'est-elle améliorée ? Non. Les peuples ont-ils un gouvernement révolutionnaire qui incarne leurs aspirations démocratiques et leurs revendications économiques ? Non plus. (Salah Sakhri, alterinfo.net 17/05/2013)

... les mouvements de résistance et de révolte n'ont pas vraiment de projet réel, ils sont émiettés et manquent de stratégie et d'objectifs communs. (Samir Amin 2012)

Les raisons de ce manque de consensus se retrouvent aussi dans l'adjectif dénominatif " arabe ». Le toponyme " arabe » est en effet un lieu d'ambiguïté et de " conflits d'interprétation " puisque " doté d'une polyréférentialité impliquant sa polysémie" (Paveau, 2008: 23) ; en d'autres mots, on comprend qu'" arabe " ne renvoie pas seulement à une dénomination géographique, "mais dessine des cheminements sémantiques complexes, contingents et parfois originaux, à travers les cadres culturels, identitaires, affectifs et mémoriels d'un sujet ou d'un groupe "(ibid). De plus, la dénomination d'un toponyme "se teinte d'idéologie à l'encontre de toute systématique, au hasard des alliances, de ce qui est censé être " politiquement correct » ou, au contraire, des inimitiés géopolitiques. [...] La dénomination d'un toponyme n'est pas neutre politiquement [...] C'est que les noms de lieux subissent une influence qu'on peut qualifier de "poids de l'histoire " (Grass, 2006). Loin d'être un simple " automatisme » (Schapira, 1999: 1), l'emploi de l'expression PA semble être un choix conscient délibéré. Par l'ajout de l'adjectif classifiant " arabe ", l'expression révèle la reproduction d'imaginaires géographiques stéréotypés, d'images se renforçant les unes les autres. « L'image d'un "monde arabe" perçu erronément comme une aire qui aurait une unité culturelle et politique certaine » (Oiry-Varacca, 2012) :

Beaucoup de nos contemporains ont des difficultés à percevoir l'évolution du monde au-delà de leur propre existence. Ayant toujours connu l'Europe démocratique et un monde arabo-musulman sous-développé et despotique, l'intuition générale a tendance à percevoir les choses comme immuables, et les pays démocratiques destinés à le rester, et les pays non démocratiques destinés, sauf exception, à demeurer despotiques. (Philippe Fabry Le Blog de décembre 2015)

La possibilité que les peuples arabes se soulèvent et réclament leur droit à la démocratie, sans que l'Occident s'en mêle, ne pouvait donc lui traverser l'esprit (en parlant de Netanyahou $)^{6}$ (Avi Shlaim, Mouvements 2011)

Edward Saïd (1997) a bien montré comment se dessinent des rapports de domination derrière la reproduction de tels clichés, dans des contextes postcoloniaux. (Mari OiryVaracca, L'Espace Politique 06/12/2012)

Quelles sont les caractéristiques politiques, ethniques, linguistiques et religieuses des sociétés arabes ? Pourquoi leurs similitudes mettent-elles en exergue des valeurs négatives : la corruption, le despotisme, le manque de liberté d'expression et le manque de justice sociale? (N. Vahab, revue.org 2011)

Pourtant, le monde arabe porte en son sein une réelle diversité si bien qu'il convient d'éviter toute réduction sémantique et idéologique :

" Il est toujours dangereux de généraliser en parlant du « monde arabe », en ignorantla diversité des conditions objectives qui caractérisent chacun des pays de ce monde ». (Samir Amin 2011)

du Golfe Persique à l'océan Atlantique, on s'identifie au monde arabe en insistant sur les liens religieux, linguistiques et historiques communs. D'autres similarités existent, 
sans qu'elles soient forcément négatives (...) mais, elles « ne constituent certainement pas des facteurs d'union entre ces pays, et encore moins des éléments d'homogénéité entre eux. (Nader Vahab, Revue.Org 2011)

En outre, des journalistes et analystes se sont focalisés sur le terme » Maghreb » pour désigner autrement cette partie du monde, mais, très vite, les réactions furent tout aussi hostiles. Nombres de chercheurs et de militants amazighs refusent l'expression la considérant comme étant «en contradiction avec la réalité existante et vécue, ne prenant pas en considération les spécificités des populations maghrébines, et excluant les autres composantes culturelles, notamment amazighes» (Mari Oiry-Varacca L'Espace Politique, 2012).

En outre, l'expression écarterait de fait les autres pays de l'Orient ne faisant partie géographiquement pas de ces pays du Maghreb. D'autres, proposeront alors, pour éviter toute ambiguïté, des expressions telles que « pays du Nord-ouest de l'Afrique » et "pays de la péninsule arabique », ou encore "Méditerranée occidentale », "Afrique occidentale $» . .$.

Si l'expression ne fait pas l'unanimité c'est, aussi en rapport avec les contextes et conséquences politico-sociales de chaque pays. Les chemins que les divers pays ont parcourus depuis novembre 2010, fortement tributaires de leurs passés respectifs, ne mènent pas nécessairement à une « Rome démocratique, ni même aux gîtes d'étape qui en jalonnent le trajet » (Eberhard Kienle 2012).

«La redistribution des cartes dans le monde arabe connaît de sérieuses déconvenues (...) : «La Syrie, pays central du Levant, s'enlise dans une guerre communautaire (...). La Libye est menacée par les guerres tribales. L'Égypte voit la rivalité entre l'armée et les Frères musulmans s'envenimer. (...) La Tunisie apprend difficilement les codes démocratiques. L'Algérie, enfin, poursuit sa glaciation.» (Naoufel Brahimi, El Mili 2012)

Les particularités locales ont pris le pas sur les espoirs universels. L'arrivée au pouvoir des islamistes en Tunisie et en Égypte, le chaos qui règne en Libye, et l'obstination folle du pouvoir syrien forcent au calme et à la mesure. (Le Huffington Post 01/02/2013)

La polémique concernant le choix de l'expression PA ne s'arrête pas là. Certains journalistes et blogueurs iront même jusqu'à poser la théorie du complot et de la manipulation imputés à des «mains étrangères » responsables ou du moins impliquées dans ces soulèvements qu'elles qualifient de « printemps » et « révolutions arabes » :

Ces révolutions qualifiées à l'époque de populaires ont en réalité été pilotées à distance par les États-Unis (François Burgat atlantico.fr 13 Mai 2013)

«Il est clair que ce ne sont pas les États-Unis qui ont fait cette révolution, mais il n'en demeure pas moins que ce sont eux qui ont accompagné et encadré les principaux activistes que ce soit en Tunisie, en Égypte et dans les autres pays arabes à travers leur formation et ce bien avant le début de la contestation ». (Ahmed Bensaada égalité et réconciliation 25/01/2015)

Mais bien que les revendications de la rue arabe soient réelles, des recherches fouillées ont montré que les jeunes manifestants et cyberactivistes arabes étaient formés et financés par des organismes américains spécialisés dans l'«exportation» de la démocratie, tels que l'USAID, la NED, Freedom House ou l'Open Society du milliardaire George Soros, et tout cela, des années avant l'immolation par le feu de Mohamed Bouazizi. (blog Bensaada 22/01/2016)

Même à travers la caricature le PA est vu comme un complot ourdi par l'oncle Sam : 


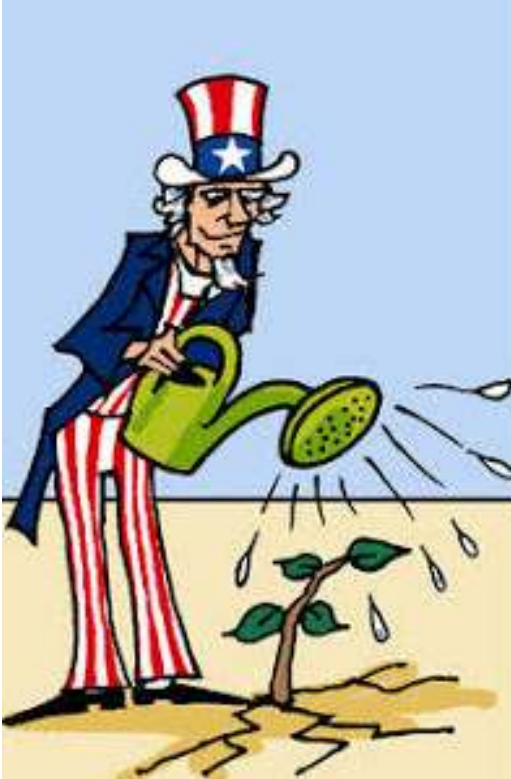

ARAB SPRING

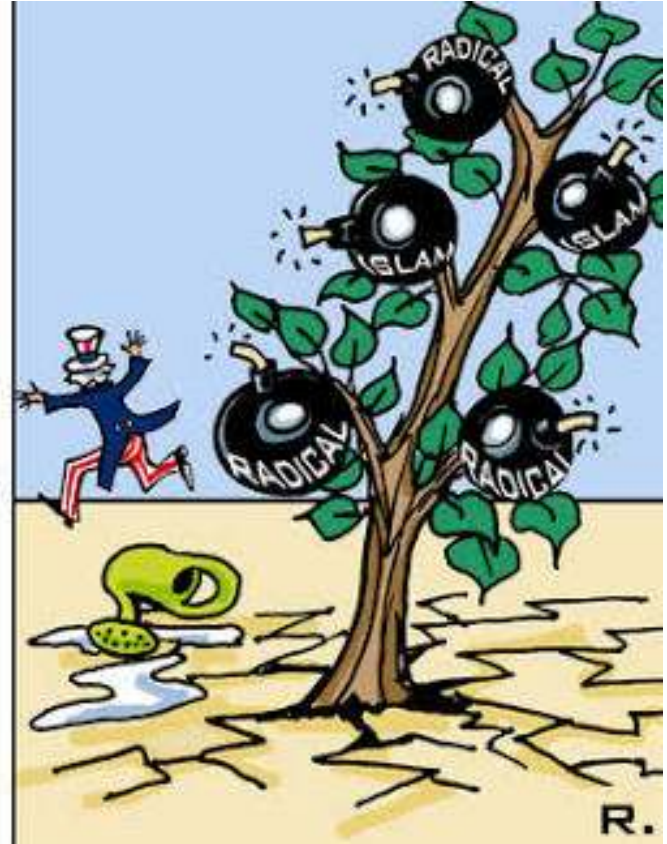

R.

Rachel Gold

Ainsi nous avons pu voir qu'en dépit de sa mise en circulation à travers des discours hétérogènes (les exemples explorés ci-dessous) l'unité lexicale PA, de par la mise en cause de sa signification, revêt un caractère problématique assez controversé.

\section{La mise en circulation de la formule en discours}

Pour saisir les phénomènes d'inscription discursive et de circulation de la formule PA, au lieu d'exploiter notre corpus dans sa totalité - vu sa diversité - nous avons opté pour une consultation de journaux français et autres algériens (d'expression française) archivés dans la base de données PressDisplay. Un tel choix nous a semblé plus aisé pour rendre compte quantitativement de la fréquence d'emploi de la formule PA.

PressDisplayest une base de données qui permet de lire les deux derniers mois de plus de 600 titres de presse française et internationale au format PDF (photos et mise en page identiques à la version papier). Les sources qui ont été consultées suite à notre interrogation de la base de données concernent les journaux: Le Monde, Le Figaro, Libération, El Watan, et le Quotidien d'Oran. Nous avons inséré le mot printemps dans le formulaire de recherche et nous avons obtenu quatre mille deux cent cinquante-six (4256) résultats ou occurrences témoignant de la fréquence d'emploi de la formule Printemps Arabe. Grâce à l'histogramme, nous remarquons nettementla section temporelle où la formule PA connaît une fréquence accrue, à savoir les années 2012-2013:

Rappelons, en effet, que des années plus tôt, à partir de 2005 précisément, l'expression PA avait déjà fait son apparition et était alors investie formellement dans des discours politiques et médiatiques pour faire référence à quelques événements ayant ébranlé certains pays du Moyen-Orient, tels que l'invasion de l'Irak, la guerre du Liban, etc. À cette époque, même si la séquence PA est très rarement utilisée (27 fois), nul doute que celle-ci fonctionne dès lors d'une façon assez singulière. Autrement dit, la séquence 
avait déjà pris « un tournant » et est devenue un enjeu (Krieg, 2009 : 85). Cependant, ce n'est qu'en 2011, que PA va refaire surface et voir son emploi s'intensifier, l'élevant ainsi au rang de formule. Krieg-Planque avance l'hypothèse selon laquelle «une fréquence accrue est une des manifestations de la notoriété du signe, et par conséquent un des indices du fait que le signe se constitue en référent social - donc possiblement en formule $(2003: 232)$.

Le pic de fréquence observé sur l'histogramme correspond aux vagues de soulèvements qui ont suivi la révolution tunisienne. Les médias s'emballent et on passe alors de révolution tunisienne à printemps tunisien puis à printemps arabe. La formule PA est alors happée, exploitée, et très souvent remise en question par ses usagers à travers la presse et autres supports médias comme vue dans les exemples étudiés plus loin.

$\mathrm{Au}$ terme de notre réflexion, nous pouvons dire que le parcours confus de l'expression PA auquel nous nous sommes intéressés nous a amené à confirmer le statut formulaire de cette expression. "Printemps arabe " est une formule puisqu'elle présente une forme figée, elle est un référent social à un certain moment, elle désigne un événement courant (historique) et par les positionnements que les acteurs-locuteurs prennent sur elle (pour ou contre), elle représente un réel objet de polémique.

Nous avons pu voir aussi, à travers des discours médiatiques et médiatisés, qu'à un moment donné, l'éclosion de ce syntagme et sa mise en circulation ont fait passer PA d'un emploi relatif à un "emploi absolu» dont le référent est problématique. Un passage, comme dirait Maingueneau (2014 : 99) «inintelligible si on ne le rapporte pas au jeu des forces, aux intérêts, aux stratégies qui à un moment donné ont rendu à la fois nécessaire et problématique l'usage d'une telle formule ». Ainsi, face à l'événement historique qui a touché certains pays arabes en 2011, l'expression PA chargée d'enjeux politiques, culturels, identitaires a permis à ses utilisateurs de se positionner politiquement dans le débat public.

Si la formule PA, ressurgie lors des événements historiques qui ont bouleversé certains pays du Maghreb et du Proche-Orient de 2011 à 2014, a connu une certaine notoriété et son heure de gloire, il s'avère qu'à l'heure actuelle, l'usage de ce syntagme semble décroître. Le printemps arabe risque-t-il de tomber en désuétude ? Le PA s'est-il arrêté en Syrie ?

D'autres syntagmes continuent de surgir aujourd'hui et à s'imposer dans cette région du monde, au gré des discours qui les supportent, participant ainsi à l'Histoire, à l'instar de " conflit syrien », « menace terroriste », « crise migratoire » ...

\section{BIBLIOGRAPHIE}

Brahimi El-Mili, N. 2012. «Le printemps arabe ; une manipulation ?», Paris : Max Milo Editions.

Calabrese Steimberg, L. 2013. La nomination des événements dans le discours d'information : entre déférence et activité collective. In Dire l'événement. Langage mémoire société. Paris : Presses Sorbonne Nouvelle, 354 pp. 
Camau, M. «Un printemps arabe ? L'émulation protestataire et ses limites », L'Année du Maghreb, VIII | 2012, consulté le 03 janvier 2014. journals.openedition.org/anneemaghreb/

Cherkaoui, M. « L'État et la Révolution. Logique du pouvoir monopoliste et mécanismes sociaux dans L'Ancien Régime de Tocqueville », Revue européenne des sciences sociales, XLI-126 | 2003, consulté le 19 juillet 2015.revues.org/

Grass, T. 2006. « La traduction comme appropriation : le cas des toponymes étrangers ». In Meta: journal des traducteurs/Meta: Translators' Journal, vol. 51, n 4, 660-667.

Krieg-Planque, A. 2009. La notion de " formule » en analyse du discours. Cadre théorique et méthodologique. Besançon : Presses Universitaires de Franche-Comté.

Krieg-Planque, A. 2003. «Purification ethnique ». Une formule et son histoire. Paris : CNRS éditions, $528 \mathrm{p}$.

Krieg-Planque, A. 2012. Analyser les discours institutionnels. Paris : A. Colin, coll. icom, série Discours et communication, $238 \mathrm{p}$.

Lugan, B."Il n'y a pas eu de Printemps Arabe !", Synthèse nationale, synthesenationale.hautetfort.com/archive/20 11/11/21. Consulté le 05 février 2012. Maingueneau, D. 2014. « Discours et analyse du discours. Une Introduction », Paris : A. Colin, 215p. Moirand, S. 2007. « Les discours de la presse quotidienne. Observer, analyser, comprendre », Paris : PUF. Oiry-Varacca, M. « Le "printemps arabe" à l'épreuve des revendications amazighes au Maroc ", L’Espace Politique, consulté le 05 juin 2018. journals.openedition.org/espacepolitique/

Paveau, M.A. 2008. « Le toponyme, désignateur souple et organisateur mémoriel. L'exemple du nom de bataille ». In Mots. Les langages du politique $\mathrm{n}^{\circ} 86$.

Schapira C. 1999. " Les stéréotypes en français : proverbes et autres formules ». Paris : Ed Ophrys.

\section{NOTES}

1. Dans son article «Printemps, révoltes et réseaux » François-Bernard Huyghe (2013) parle aussi de révolution 2.0

2. Krieg s'étant inspirée elle-même des travaux de de Fiala et Ebel (1983) lesquels ont emprunté la notion de formule à J-P Faye (1972).

3. Le concept d'" emballement médiatique " connaît aujourd'hui un essor fulgurant et un usage répandu dans la presse notamment avec les travaux de Pascal Froissart (2012).

4. La dénomination officielle de la révolution tunisienne a été acceptée par l'Assemblée nationale du pays en 2012 comme « Révolution de la liberté et de la dignité, 17 décembre 2010 - 14 janvier 2011 ».

5. Hungtington, S.cité par Mohamed Cherkaoui in «L'État et la Révolution. Logique du pouvoir monopoliste et mécanismes sociaux dans L'Ancien Régime de Tocqueville », Revue européenne des sciences sociales 30/11/2009

6. Netanyahou critique l'Occident pour avoir accordé une "exemption démocratique" à des Arabes trop immatures pour la démocratie et dont l'héritage islamique n'aurait de toute façon pas été compatible avec celle-ci.» (Avi Shlaim Israël, les États-Unis et le printemps arabe, Mouvements 2011/2 ( $\left.{ }^{\circ} 66\right)$, p. 135-144 


\section{RÉSUMÉS}

L'expression « printemps arabe » désignant les mouvements qui éclatent en 2011 en Tunisie puis dans d'autres pays, va occuper, dès son apparition, une place considérable dans les discours politiques, médiatiques et publics. De son statut de mot événement, l'expression accède à une nouvelle fonction dans le discours : celle de formule. Inspirée des travaux de Krieg-Planque sur la formule en discours, la perspective proposée dans cet article est d'interroger la notion de " printemps arabe » et suivre son destin formulaire lié à l'ordre événementiel et à son interprétation à travers l'espace discursif auquel elle participe.

The expression of "Arab Spring" designating the movements that broke out in 2011 in Tunisia and then in other Arab countries, has occupied a considerable place in political, media and public speeches. From its status as a neologism, the expression accesses a new function in the discourse : that of formula. Inspired by the works of Alice Krieg-Planque on formula in discourse analysis, the perspective proposed in this article is to question the notion of "Arab Spring" and follow the change in its discursive formula related to the event and its interpretation through the discursive space in which it participates.

\section{INDEX}

Mots-clés : printemps arabe, formule, figement, polémique, analyse de discours

Keywords : Arab Spring, formula, congestion, controversy, discourse analysis

\section{AUTEURS}

\section{FARID BENRAMDANE}

Université de Mostaganem, Algérie 